\title{
Ge/SiGe parabolic quantum wells
}

\author{
Andrea Ballabio ${ }^{1}$, Jacopo Frigerio ${ }^{1}$, Saleh Firoozabadi ${ }^{2}$, Daniel \\ Chrastina $^{1}$, Andreas Beyer ${ }^{2}$, Kerstin Volz ${ }^{2}$, Giovanni Isella ${ }^{1}$ \\ ${ }^{1}$ L-NESS, Department of Physics, Politecnico di Milano, Via Anzani 42, 22100 \\ Como, Italy \\ 2 Materials Science Center and Faculty of Physics, Philipps-Universität Marburg, \\ Hans-Meerweinstraße 6, 35032 Marburg, Germany \\ E-mail: andrea.ballabio@polimi.it
}

\begin{abstract}
Quantum wells with a parabolic confining potentials allows the realization of semiconductor heterostructures mimicking the physical properties of a quantum harmonic oscillator. Here we report the attempt of attaining such parabolic quantum wells (PQWs) within the Ge/SiGe material platform. Multiple PQWS featuring different widths and composition have been epitaxially grown and characterized by means of high-resolution X-ray diffraction and scanning transmission electron microscopy. The compositional profile is seen to deviate slightly from an ideal parabola, but the quantum confined states are almost equally spaced within the valence and conduction band as indicated by photoreflectance measurements and $\mathbf{k} \cdot \mathbf{p}$ modelling. Keywords: Silicon germanium, quantum wells, quantitative STEM
\end{abstract}

Submitted to: J. Phys. D: Appl. Phys. 


\section{Introduction}

Germanium and $\mathrm{Si}_{1-x} \mathrm{Ge}_{x}$ heterostructures have been widely investigated as a means to integrate active photonic devices such as photodetectors [1] and optical modulators [2] on silicon. $\mathrm{Ge} / \mathrm{Si}_{1-x} \mathrm{Ge}_{x}$ quantum wells (QWs) are suitable for the realization of optical modulators, exploiting the quantum-confined Stark effect (QCSE) to achieve intensity or phase modulation [3], even within a waveguide integrated platform [4]. Most of these applications exploit multiple "square" quantum wells (SQWs), however more efficient phase modulation, intersubband photodetectors [5] or non-linear effects could be obtained if more complicated QW structures, such as asymmetric coupled QWs [6, 7] or parabolic QWs, were available.

In parabolic quantum wells (PQWs) the confining potential mimics the well-known harmonic oscillator problem, giving rise to multiple resonant electronic states that could enhance optical non-linearities [8-10].Moreover, it has been shown that even intersubband detectors could benefit from a parabolic confining potential, since the ground state would be strongly confined by relatively wide barriers, resulting in low dark current, while minibands could be formed from excited states [11]. Polarization independent integrated optical switches based on QCSE have also been demonstrated by exploiting PQWs $[12,13]$. PQWs also find applications in light emitting devices: $\mathrm{THz}$ radiation has been detected from subband recombination in AlGaAs PQWs [14], and the nature of the subband states in the PQW has been considered for the fabrication of lasers, exploiting bosonic condensation of excitons [15-17].

All previous experimental work on PQWs relied on III-V compound semiconductors [18]. Moreover, due to the non-trivial growth procedure, associated with the fine control over the flux of precursors, in many works the parabolic compositional profile has been approximated with an "equivalent" PQW profile realized as a superlattice $[14,19,20]$. These kinds of structures, which are usually deposited by molecularbeam epitaxy, are not appealing for industrial application, due to the complex and time-consuming growth procedure.

The growth of PQWs in the $\mathrm{Si}_{1-x} \mathrm{Ge}_{x}$ material system has not yet been demonstrated, but it can be promising for the integration on $\mathrm{Si}$ of detectors, modulators, and non-linear media operating in the mid-infrared, a spectral range where $\mathrm{Si}_{1-x} \mathrm{Ge}_{x}$ heterostructures have already shown a great potential for sensing applications [21]. This motivated our effort to epitaxially grow $\mathrm{Si}_{1-x} \mathrm{Ge}_{x} \mathrm{PQWs}$ and to characterize them by means of high resolution X-ray diffraction (HR-XRD), and photo-reflectance (PR) spectroscopy. In addition, $Z$-contrast high angle annular dark

field scanning transmission electron microscopy (HAADF-STEM) is applied to quantify the composition of $\mathrm{Si}_{1-x} \mathrm{Ge}_{x}$ QWs at an atomic scale, applying a procedure similar to [22-25]. Our work indicates that, even though the concentration profile deviates from an ideal parabola, the optical properties of such heterostructures differ from those of conventional SQWs, with energy levels which are almost equally spaced. 


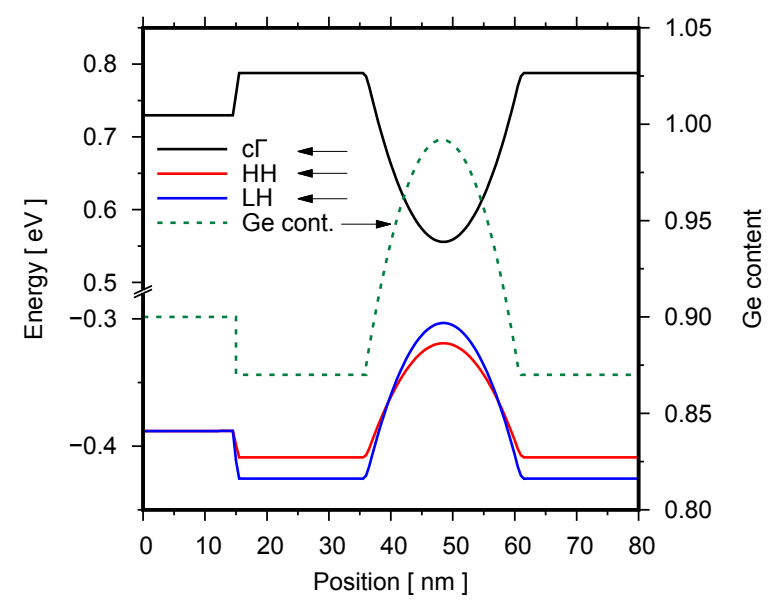

Figure 1. Schematic representation of the Ge content profile, and resulting bandalignment, in an ideal SiGe PQW.

\section{Epitaxial growth}

The nominal Ge content profile for the case of one PQW, embedded between two $\mathrm{Si}_{0.13} \mathrm{Ge}_{0.87}$ barriers and grown on a relaxed $\mathrm{Si}_{0.1} \mathrm{Ge}_{0.9}$ buffer, is shown in figure 1 . The compositional profile has been designed to achieve strain compensation between the compressively-strained central part of the PQW (which contains more Ge than the relaxed buffer) and the tensile-strained regions comprising the barrier and the lateral parts of the PQW. Even though a parabolic compositional profile does not strictly provide a parabolic confining potential, the deformation potential modelling of the conduction band edge at the $\Gamma$ point $(\mathrm{c} \Gamma)$ and for the heavy-hole $(\mathrm{HH})$ and lighthole (LH) bands (also shown in figure 1) do not significantly deviate from a parabola. Therefore in the following the small difference between the nominal compositional profile and the nominal confining potential will be neglected.

A set of PQW heterostructures, with varying QW thickness, barrier thickness, and barrier composition $\left(t_{Q W}, t_{B}\right.$, and $x_{B}$ respectively) have been grown and characterized together with an additional SQW heterostructure for comparison. The relevant structural properties of the analysed samples are summarized in table 1. The nominal concentration at the parabola vertex (and in QW region of the SQW structure) is 100\% Ge in all cases. The heterostructures actually consist of multiple QW/barrier periods repeated between 10 and 50 times for the samples listed in table 1.

All samples have been deposited on $100 \mathrm{~mm} \mathrm{Si(001)}$ wafers by means of low-energy plasma-enhanced chemical vapour deposition (LEPECVD) [26].

The relaxed $\mathrm{Si}_{1-x} \mathrm{Ge}_{x}$ buffer is obtained by linearly increasing the Ge content with a grading rate of $7 \% / \mu \mathrm{m}$ from zero to a final composition ranging from $x \simeq 0.79$ to 0.93 for the different samples reported in table 1. Full relaxation is achieved by growing a $2 \mu \mathrm{m}$ thick $\mathrm{Si}_{1-x} \mathrm{Ge}_{x}$ layer on top of the graded part, to form a virtual substrate (VS). During the relaxed buffer deposition the growth rates varies between 5 and $10 \mathrm{nms}^{-1}$ and the substrate temperature is progressively reduced from $760^{\circ} \mathrm{C}$ to $450^{\circ} \mathrm{C}$ in order to suppress 
strain-induced surface roughening. Typical RMS roughness and dislocation densities are $2.8 \mathrm{~nm}$ and $3 \times 10^{6} \mathrm{~cm}^{-3}$, respectively [27]. During the multiple QW/barrier growth step the deposition rate is reduced to $\sim 0.55 \mathrm{~nm} / \mathrm{s}$ to better control the compositional profile, obtained by gradually changing the precursor gas flows $\left(\mathrm{SiH}_{4}\right.$ and $\left.\mathrm{GeH}_{4}\right)$ by means of calibrated mass flow controllers.

Table 1. Structural properties of the of the analysed PQW and SQW samples. For each sample the QW thickness $\left(t_{\mathrm{QW}}\right)$ in $\mathrm{nm}$, barrier thickness in $\mathrm{nm}\left(t_{\mathrm{B}}\right)$ and barrier composition $\left(x_{\mathrm{B}}\right)$, relaxed buffer composition $\left(x_{\mathrm{VS}}\right)$, and number of periods are indicated.

\begin{tabular}{ccccccc}
\hline & \multicolumn{5}{c}{ PQW } & SQW \\
& A & B & C & D & E & \\
\hline$t_{\mathrm{QW}}$ & 25 & 47 & 62 & 21 & 37 & 17 \\
$t_{\mathrm{B}}$ & 22 & 46 & 41 & 14 & 20 & 32 \\
$x_{\mathrm{B}}$ & 0.87 & 0.87 & 0.85 & 0.655 & 0.7 & 0.85 \\
$x_{\mathrm{VS}}$ & 0.93 & 0.90 & 0.92 & 0.80 & 0.79 & 0.90 \\
Periods & 20 & 20 & 10 & 15 & 15 & 50 \\
\hline
\end{tabular}

\section{Structural characterization}

HR-XRD measurements have been performed on all samples with a PANalytical X'Pert PRO MRD diffractometer. The (004) and (224) reciprocal space maps (RSM) of sample PQW-E are shown in figure 2(a) and (b), respectively. A clear superlattice period is observed indicating regular periodicity of the multiple PQW structure, and the effective strain compensation of the PQW with respect to the VS. Figure 2(c) shows the (004) $\omega-2 \theta$ scan, together with multi-beam dynamical Darwin model simulations [28, 29] as implemented in xrayutilities [30]. In the inset of figure 2(c) the PQW profiles used for the simulations are reported. By assuming an ideal parabolic profile (see figure 2 blue line) it is possible to fit the low order peaks, however the intensity of higher-order peaks (at $q_{\perp}<7.0 \mathrm{~nm}^{-1}$ ) poorly fits the experimental data.

For these reasons we carried out transmission electron microscopy (TEM) measurements on PQW-E and on the reference SQW sample. A MultiPrep ${ }^{\mathrm{TM}}$ System was used in combination with a Model 691 precision Ar-based ion polishing system, to obtain an electron transparent sample with a thickness less than $50 \mathrm{~nm}$. A doubleaberration-corrected JEOL 2200FS scanning transmission electron microscope operating at $200 \mathrm{kV}$ was used to extract the chemical composition of the $\mathrm{Ge} / \mathrm{Si}_{1-x} \mathrm{Ge}_{x}$ QWs at atomic resolution, using the high-angle annular dark field (HAADF) technique. The incident electron probe's convergent semi-angle was $21.3 \mathrm{mrad}$. In addition, a JEOL EM24590YPDFI annular dark field detector was used to detect the scattered electrons in the range of 66-266 mrad and 68-270 mrad for samples SQW and PQW-E, respectively. In order to decrease the noise and also avoid the effect of sample drift during the 

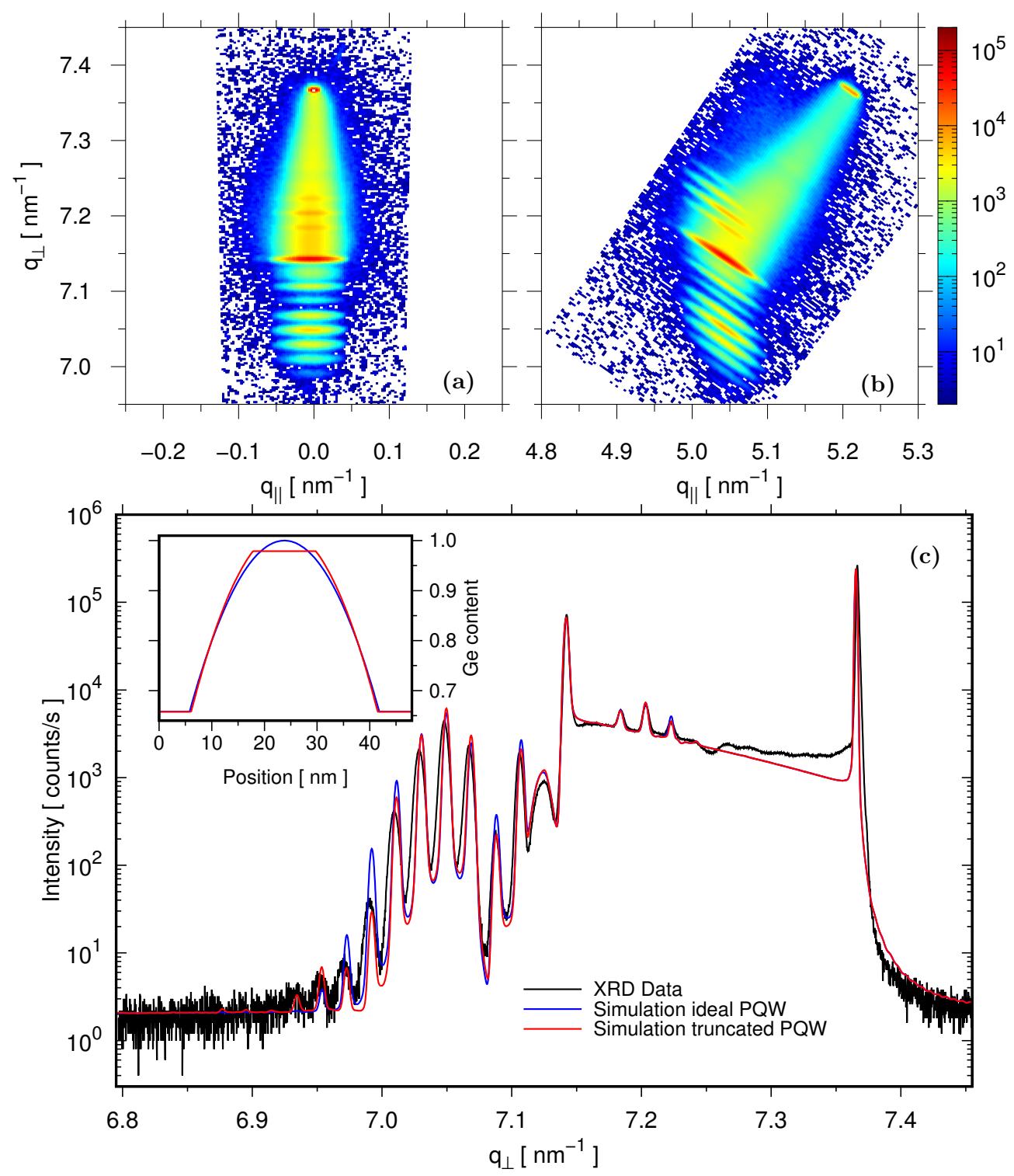

Figure 2. HR-XRD measurements of sample PQW-E. (a) Symmetrical and (b) asymmetrical reciprocal space maps, taken around the (004) and (224) peaks respectively, show that the buffer is fully relaxed and that the periodic PQW structure is lattice-matched to the final composition of the VS. (c) A detailed $\omega-2 \theta$ scan through the (004) reflection can be compared to the results of dynamical simulations for the ideal and truncated PQW profiles shown in the inset. The truncated PQW profile provides a satisfactory fit even at higher-order satellite peaks, which is not true of the best ideal PQW fit.

measurement, every image is an average of ten measured images non-rigidly aligned using the Smart-Align software package [31].

An accurate evaluation of the compositional profile of the Ge concentration across the QWs can be achieved by high-angle annular dark field (HAADF) scanning transmission electron microscopy (STEM). The amount of Rutherford scattered electrons used to obtain HAADF-STEM images has a strong dependence on atomic 


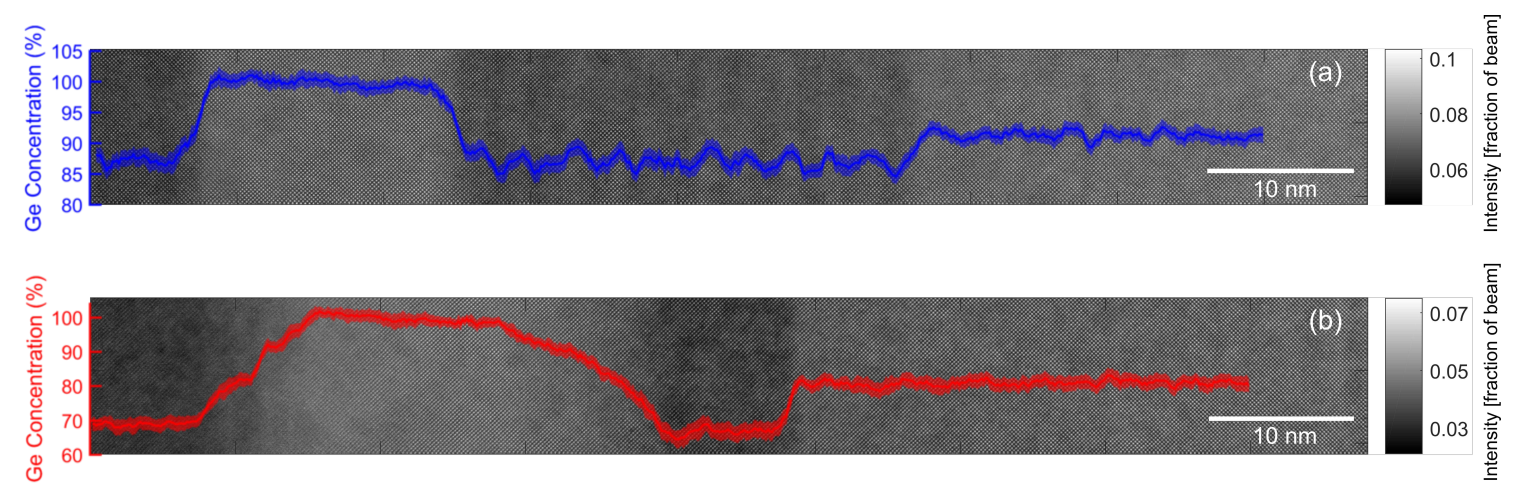

Figure 3. HAADF-STEM images of the samples (a) SQW with "square" QWs and (b) PQW-E with parabolic QWs. The intensities are normalized to the impinging beam. In addition, assuming the virtual crystal approximation, the images are calibrated based on the lattice constant of the buffer layer (visible on the right of the images) calculated from average concentrations measured from HR-XRD. The average concentration profile in every lattice plane perpendicular to the growth direction of the SQW and PQW-E samples is superimposed onto their respective HAADF-STEM images.

number. Thus, the resulting images are sensitive to the chemical composition of the material, i.e. heavier atoms of Ge cause higher intensity in an image of a $\mathrm{Ge} / \mathrm{Si}_{1-x} \mathrm{Ge}_{x}$ structure. The atomic resolution HAADF-STEM measurements of the SQW and PQWE samples in the [010] direction are shown in figure 3(a) and figure 3(b), respectively. The growth direction in both measurements is from right to left starting from a buffer layer, showing the barrier and then the first QW. As can be seen in the both images, the lower concentration of $\mathrm{Ge}$ in the barrier causes lower intensity in both samples. In order to quantitatively analyse the chemical composition of Ge in every atomic column, it is necessary to compare the measured images with the complementary contrast simulations obtained from the software package STEMsalabim [32]. The determination of the concentration is explained in detail in [33]. Due to the dependence of the HAADF-STEM intensity on the sample thickness in addition to atomic number, it is necessary to determine the spatial dependence of the sample thickness. Since the average concentration of Ge in the VS is known from HR-XRD, $\mathrm{Si}_{1-x} \mathrm{Ge}_{x}$ layers with the specified concentration can be simulated and the resulting images can be compared with the measured images in order to locally determine the thickness. This thickness map can then be used to obtain the Ge concentration of each atomic column. The profiles of Ge concentration in every lattice plane perpendicular to the growth direction in both samples are illustrated as superimposed curves in figure 3(a) and 3(b), respectively.

The nominal widths of the first barrier and QW were 32 and $17 \mathrm{~nm}$ for the SQW sample, and 10 and $37 \mathrm{~nm}$ for PQW-E (in the case of PQW-E the barrier before the first PQW was half the width, nominally $20 \mathrm{~nm}$, of the barriers between each PQW). Although fluctuations are clearly visible in the concentration profiles, the averaged values closely match the Ge concentration obtained from HR-XRD. The HR-XRD measurements of the SQW sample match with dynamical simulations specifying Ge 
concentrations of $84.0 \%$ and $98.5 \%$ in the barriers and QWs, respectively, with widths of 30.5 and $18.5 \mathrm{~nm}$. In figure 2 the simulated ideal PQW profile finds $65.8 \%$ in the barriers (width $16.0 \mathrm{~nm}$, so a first barrier width of $8.0 \mathrm{~nm}$ ) and $100 \%$ at the highest point of the QWs (total PQW width $36.0 \mathrm{~nm}$ ).

In the SQW, intermixing is visible at both bottom and top interfaces to a similar degree, indicating the absence of strain induced roughening typical of $\mathrm{Si}_{1-x} \mathrm{Ge}_{x}$ heteroepitaxy [34]. Although the Ge content profile of the PQW substantially differs from that of the SQW, it does not completely match that of a parabola. In particular the high Ge-content part appears to be flattened, to form a region with an almost constant Ge content of $x \simeq 0.98$. To validate the statistical relevance of the Ge profile extracted from the STEM analysis of figure 3 we have modelled the HR-XRD spectra of figure 2(c) by assuming a truncated parabolic profile (see figure 2(c) red line). In this case the modelling nicely reproduces also the high-order superlattice peaks, thus confirming the STEM analysis. In figure 2 the simulated truncated PQW profile finds $65.8 \%$ in the barriers (width $16.6 \mathrm{~nm}$ ) and $97.9 \%$ in the highest point of the QWs (total PQW width $35.4 \mathrm{~nm})$.

\section{Transition energies and absorption coefficient calculations}

An eight-band $\mathbf{k} \cdot \mathbf{p}$ method implemented in nextnano [35] has been used to calculate the energy levels around the $\Gamma$ point of the conduction and valence bands. The intensity of the optical interband transitions has been calculated, including excitonic effects, by means of an energy-minimization approach [36, 37]. The relevant deformation potentials are shown in table 2 and Luttinger parameters have been taken from [38]. An average valence band offset of $\Delta \mathrm{E}_{\mathrm{v}}^{\mathrm{Av}}=0.8 \mathrm{eV}$ has been used in agreement with ab-initio calculations of [39] and experimental data in [40]. The compositional profile and strain

levels required as an input for the $\mathbf{k} \cdot \mathbf{p}$ calculations have been extracted from the HR$\mathrm{XRD}$ and STEM analyses presented in the previous section.

Table 2. Relevant deformation potentials (in eV) for Si and Ge.

\begin{tabular}{ccc}
\hline & $\mathrm{Si}$ & $\mathrm{Ge}$ \\
\hline$a_{\mathrm{c}}^{\Gamma}$ & $-10.39^{a}$ & $-10.41^{a}$ \\
$a_{\mathrm{v}}$ & $1.80^{b}$ & $1.24^{b}$ \\
$b$ & $-2.10^{c}$ & $-1.88^{d}$ \\
$E_{\mathrm{g}}^{\Gamma}$ at RT & $4.2^{e}$ & 0.80 \\
\hline a) [41] b) [42] c) [43] d) [44] e) $[45]$
\end{tabular}

Figure 4(a) shows the confining potential and the ensuing energy levels at the $\Gamma$ point of the $\mathrm{CB}$ for three different confining potentials. In one case the $\mathbf{k} \cdot \mathbf{p}$ calculation has been performed using the compositional profile from the STEM data of figure 3 and assuming a pseudomorphic interface between the strained multiple PQW structure and the relaxed buffer. In a second case a parabolic best-fit of the STEM-extracted 

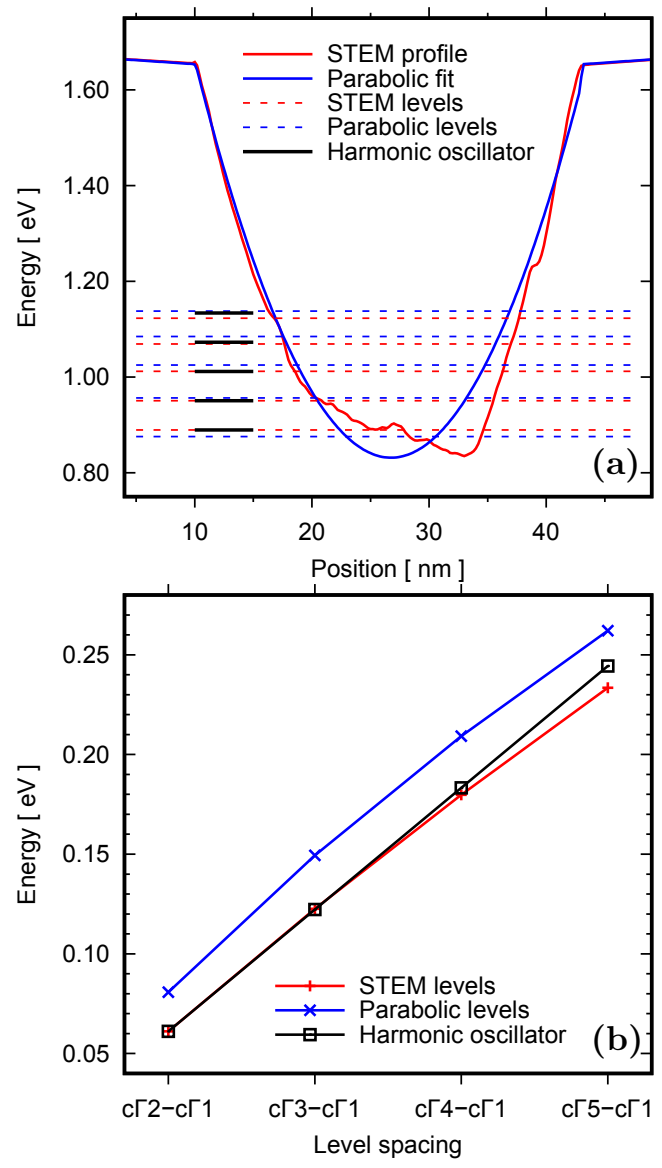

Figure 4. (a) Conduction band $(\mathrm{c} \Gamma)$ energy profile and quantum confined levels for the compositional profile extracted from figure 3 (red solid line) and a finitebarrier parabolic profile fitted to STEM profile (blue solid line) shown in panel. The energy levels corresponding to these two profiles are shown as the dashed horizontal lines. Energy levels corresponding to an ideal infinite-barrier harmonic oscillator are also shown as bold black segments. (b) Energy difference between $\mathrm{c} \Gamma 1$ and $\mathrm{c} \Gamma \mathrm{m}$ for $m=2,3,4,5$ for the three confining potentials under investigation.

profile has been used as a confining potential, thus obtaining a finite barrier harmonic oscillator. Eventually the parabolic profile has been extended to the infinite barrier case, thus recovering an ideal harmonic oscillator potential. Figure 4(a) suggests that the deviation of the STEM profile from an ideal parabolic profile does not completely spoil the regular spacing of the energy levels. This is confirmed by figure 4(b) where the energy spacing of the first four quantum confined states are compared for the case of an ideal parabolic potential, the best-fit finite barrier parabolic profile and the STEM profile. Equally spaced energy levels are obtained even in the case of a non-ideal confining potential, with small deviations due to the finite height of the barrier, for the higher quantum numbers. 


\section{Photoreflectance measurements}

In order to experimentally confirm the onset of equally spaced transitions, photoreflectance (PR) measurements [46-48] have been performed on the PQW and reference SQW sample. At variance with transmission measurements, PR gives access to transitions with energies exceeding the absorption edge of the substrates (indirect gap absorption in the graded buffer and $\mathrm{Si}$ wafer in our case). Moreover, the derivative nature of the PR signal highlights low intensity transitions such as those involving quantum confined states with different quantum numbers. The reflectivity is measured by shining a monochromatic beam on the sample surface, obtained by means of a lampmonochromator set-up (Newport ${ }^{\circledR}$ Cornerstone 260) with a typical resolution of $\sim 4 \mathrm{~nm}$. The reflected light is then collected and focused on a Thorlab ${ }^{\circledR}$ Ge DET50 detector. The reflectivity signal is acquired from a region of the sample surface simultaneously illuminated by means of a $632 \mathrm{~nm}$ laser diode with typical output power of $10 \mathrm{~mW}$, modulated by a mechanical chopper at $387 \mathrm{~Hz}$. The electric field associated with the generation and recombination of photoexcited carriers modulates the samples reflectivity which is demodulated by means of lock-in amplifier. The room temperature PR spectra of the SQW is shown in figure 5(a), which also shows the fitting curve obtained by using the PR lineshape [48] for each transition $j$ given by:

$$
\frac{\Delta R}{R}=R e \sum_{j} A_{j} e^{i \Phi_{j}}\left(E-E_{j}+i \Gamma_{j}\right)^{-k}
$$

where $A_{j}$ and $\Phi_{j}$ are the amplitude and phase of the lineshape, $E_{j}$ is the energy of the transition and $\Gamma_{j}$ is the broadening parameter. The $k$ parameter in (1) takes the value of $k=2$ in the case of excitonic transitions, $k=3$ for $2 \mathrm{D}$ critical points and $k=2.5$ for $3 \mathrm{D}$ critical points $[49,50]$.

Due to the large number of free parameters the fitting procedure has been performed using the transition energies and absorption coefficient obtained by $\mathbf{k} \cdot \mathbf{p}$ modelling as initial values. Almost all the transitions observed in figure 5 are attributed to excitonic transitions within the QW with the exception of the two features at higher energy which can be ascribed to $\mathrm{LH}-\mathrm{c} \Gamma$ and $\mathrm{HH}-\mathrm{c} \Gamma$ transitions in the strained $\mathrm{Si}_{0.15} \mathrm{Ge}_{0.85}$ barrier $[49,50]$. As an example the PR spectrum of sample $\mathrm{A}$ is shown in figure $5(\mathrm{~b})$ together with the lineshape fitting curve. Figure 6 shows a comparison between the transition energies extracted from PR measurements and those calculated by $\mathbf{k} \cdot \mathbf{p}$ modelling. For this sample the simulated structure has a $25 \mathrm{~nm}$ thick PQW with a vertex composition $x_{\mathrm{QW}}=0.992,21 \mathrm{~nm}$ thick barriers with a composition $x_{\mathrm{B}}=0.87$, and a VS composition $x_{\mathrm{VS}}=0.93$. Experiments and calculations are in very close agreement demonstrating the robustness of our analysis and the correct labelling of optical transitions. Information regarding strain and composition of the barrier can be obtained considering the values and separation of the $\mathrm{LH}-\mathrm{c} \Gamma$ and $\mathrm{HH}-\mathrm{c} \Gamma$ transitions in

the barrier [49]: the tensile strain in the barriers leads to a separation of the LH and $\mathrm{HH}$ bands, raising the energy of the $\mathrm{LH}$ and decreasing $\mathrm{HH}$ (the $\mathrm{HH}-\mathrm{c} \Gamma$ energy gap is $\left.E_{\mathrm{g}}=1.273 \mathrm{eV}\right)$. This information, combined with the Ge content of the barrier and 

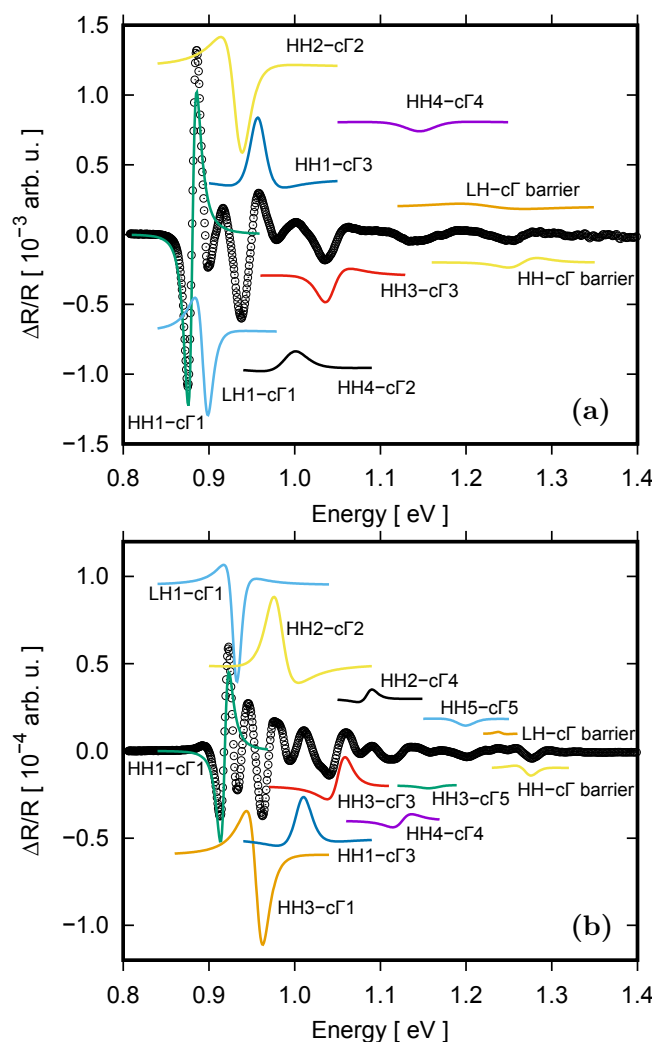

Figure 5. Photoreflectance spectra for (a) the SQW and (b) sample A. In both cases the contribution to the overall spectrum of each transitions is vertically displaced for the sake of clarity while the continuous line represents the best-fit to the experimental data (circles).

the VS extracted from the XRD, further confirms the self-consistency of the parameters used in the simulations. A similar analysis has been performed on all samples reported in table 1 and the extracted transition energies are reported in table 3 .

Table 3. Transition energies as obtained from PR measurements

\begin{tabular}{|c|c|c|c|c|c|c|}
\hline & \multicolumn{5}{|c|}{ PQW } & \multirow[t]{2}{*}{ SQW } \\
\hline & $\mathbf{A}$ & B & C & D & $\mathbf{E}$ & \\
\hline НH1-сГ1 & 0.918 & 0.877 & 0.853 & 0.992 & 0.963 & 0.880 \\
\hline LH1-cГ1 & 0.932 & - & 0.881 & 1.058 & - & 0.895 \\
\hline НН3-сГ1 & 0.958 & - & - & 1.083 & 0.979 & - \\
\hline НH2-cГ2 & 0.984 & 0.913 & 0.907 & 1.131 & 1.035 & 0.934 \\
\hline НH1-cГ3 & 1.010 & - & - & - & 1.074 & 0.957 \\
\hline НН3-сГ3 & 1.054 & 0.958 & 0.933 & 1.253 & 1.118 & 1.032 \\
\hline НH2-cГ4 & 1.089 & - & - & - & 1.144 & 1.056 \\
\hline НH4-cГ4 & 1.125 & 1.011 & 0.966 & - & 1.222 & 1.129 \\
\hline НН3-сГ5 & 1.154 & - & - & - & - & - \\
\hline НH5-сГ5 & 1.201 & 1.064 & 1.010 & - & 1.321 & - \\
\hline НH6-сГ6 & - & 1.111 & 1.048 & - & - & - \\
\hline
\end{tabular}




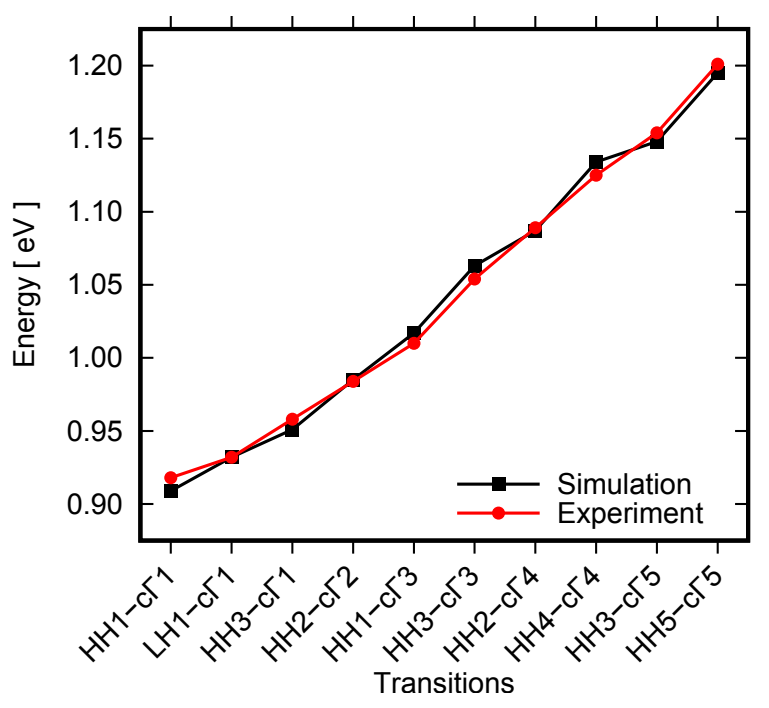

Figure 6. Comparison of the transition energies extracted from the PR measurements on sample A in figure $5(\mathrm{~b})$, and calculated by $\mathbf{k} \cdot \mathbf{p}$ modelling.

\section{Discussion}

In the case of a PQW the transition energy $E_{n, m}$ between the $n^{\text {th }} \mathrm{HH}$ state and the $m^{\text {th }}$ $\mathrm{c} \Gamma$ state can be written as:

$$
E_{n, m}=E_{\mathrm{g}}+\left(n-\frac{1}{2}\right) \Delta \mathrm{HH}+\left(m-\frac{1}{2}\right) \Delta \mathrm{c} \Gamma,
$$

where $\Delta \mathrm{HH}$ and $\Delta \mathrm{c} \Gamma$ are the $\mathrm{HH}$ and $\mathrm{c} \Gamma$ confined state energy spacings, respectively. The energies of optical transitions between states with the same quantum number $(n=m)$ should therefore be equally spaced by

$$
\Delta E_{n, m}=E_{n+1, m+1}-E_{n, m}=\Delta \mathrm{HH}+\Delta \mathrm{c} \Gamma .
$$

To benchmark our heterostructures against this fundamental property of PQWs, the data reported in table 3 have been recast in figure 7 showing the transition energies for increasing quantum numbers $n$ and $m=n$. Indeed the data points appear to follow a linear behaviour. The values of $\Delta E_{n, m}$ obtained from a least-square linear regression for all the samples under investigation are reported in table 4 together with the coefficient of correlation $R$. For the PQWs $R$ is always approaching 0.99 while it is 0.98 in the SQW case. Consequently standard deviations of a few meV are found in the case of the PQW while a value of $10 \mathrm{meV}$ is found for the SQW sample. These results indicate that, despite the non-ideal compositional profile, equally spaced energy levels are obtained in PQWs. 


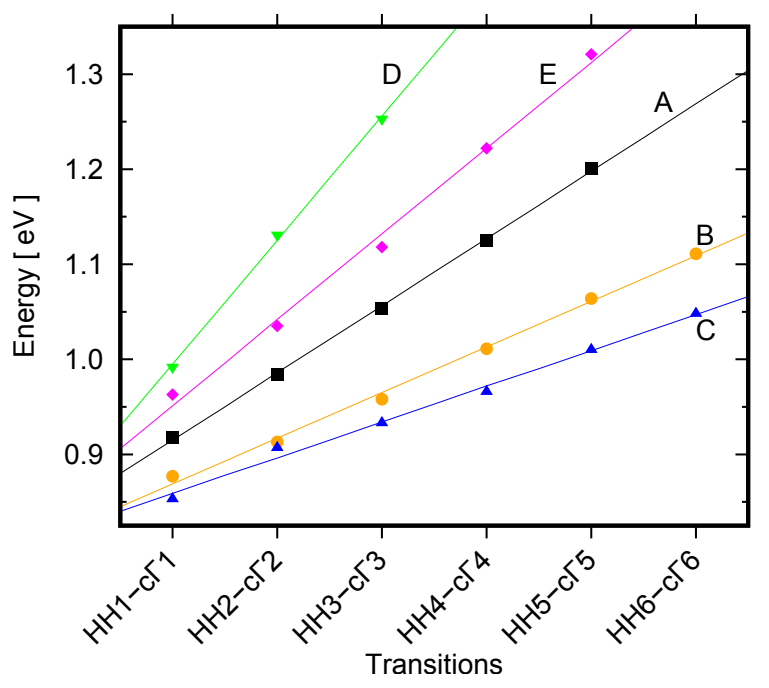

Figure 7. Energy of the transitions between quantum confined states $\mathrm{HH} n-\mathrm{c} \Gamma m$ with $n=m$ for all the PQW samples. The straight lines represent least-squares linear regressions of the experimental data.

Table 4. Summary of results obtained from the linear regression of the transition energies. $\Delta E_{n, m}$ given by (3) is the difference in energy (in meV) between the $\mathrm{HH} n$ $\mathrm{c} \Gamma m$ and $\mathrm{HH}(n+1)-\mathrm{c} \Gamma(m+1)$ transitions, for $n=m$, and the corresponding standard deviation is given in parenthesis. $R$ is the coefficient of correlation for the linear regression.

\begin{tabular}{ccccccc}
\hline & \multicolumn{4}{c}{ PQW } & & SQW \\
& A & B & C & D & E & \\
\hline$\Delta E_{n, m}$ & $70(1)$ & $48(1)$ & $37(1)$ & $131(5)$ & $89(4)$ & \\
$R$ & 0.999 & 0.997 & 0.998 & 0.998 & 0.996 & 0.985 \\
\hline
\end{tabular}

Relying solely on transitions involving quantum confined states with $n=m$ it is possible to estimate only $\Delta \mathrm{HH}+\Delta \mathrm{c} \Gamma$, however $\mathrm{PR}$ measurements give access also to transitions between states with different quantum numbers i.e. $n \neq m$. Even though the heterostructures under investigation are not perfectly symmetric the only measurable transitions are those linking two odd or two even states. In order to extract the values $E_{\mathrm{g}}, \Delta \mathrm{HH}$, and $\Delta \mathrm{c} \Gamma$ starting again from (2) we have performed least square fitting this time including both $n=m$ and $n \neq m$ transitions. In the case of the PQW sample A, values of $E_{\mathrm{g}}=0.885 \mathrm{eV}, \Delta \mathrm{HH}=0.020 \mathrm{eV}$, and $\Delta \mathrm{c} \Gamma=0.046 \mathrm{eV}$ have been obtained. The sum of the $\Delta \mathrm{HH}$ and $\Delta \mathrm{c} \Gamma$ is $67 \mathrm{meV}$, in good agreement with the linear fit reported in figure 7 and table 4 , which validates our analysis.

\section{Conclusions}

We have designed, epitaxially grown, and characterized, by means of HR-XRD and STEM, Ge/SiGe quantum wells with the aim of achieving a parabolic confining 
potential. Photoreflectance measurements indicate that the quantum confined levels are equally spaced in energy. These results are analysed using an 8-band $\mathbf{k} \cdot \mathbf{p}$ approach implemented in nextnano simulations. These results might be exploited in mid-IR intersubband detectors and for second-harmonic generation.

\section{References}

[1] Chaisakul P, Marris-Morini D, Isella G, Chrastina D, Le Roux X, Edmond S, Cassan E, Coudevylle J R and Vivien L 2011 Appl. Phys. Lett. 98131112

[2] Kuo Y H, Lee Y K, Ge Y, Ren S, Roth J E, Kamins T I, Miller D A B and Harris J S 2005 Nature 437 1334-1336

[3] Frigerio J, Chaisakul P, Marris-Morini D, Cecchi S, Roufied M S, Isella G and Vivien L 2013 Appl. Phys. Lett. 102061102

[4] Chaisakul P, Marris-Morini D, Frigerio J, Chrastina D, Rouifed M S, Cecchi S, Crozat P, Isella G and Vivien L 2014 Nature Photonics 8 482-488

[5] Gallacher K, Ballabio A, Millar R W, Frigerio J, Bashir A, MacLaren I, Isella G, Ortolani M and Paul D J 2016 Appl. Phys. Lett. 108091114

[6] Frigerio J, Vakarin V, Chaisakul P, Ferretto M, Chrastina D, Le Roux X, Vivien L, Isella G and Marris-Morini D 2015 Sci. Reports 515398

[7] Frigerio J, Ballabio A, Ortolani M and Virgilio M 2018 Opt. Express 26 3186131872

[8] Huang Y and Lien C 1994 J. Appl. Phys. 75 3223-3225

[9] Guo K X and Gu S W 1993 Phys. Rev. B 47 16322-16325

[10] Zhang L and Xie H J 2004 Physica E 22 791-796

[11] Karunasiri R P G and Wang K L 1988 Superlattices and Microstructures 4 661-664

[12] Ishikawa T, Nishimura S and Tada K 1990 Jpn. J. Appl. Phys. 29 1466-1473

[13] Tada K, Nishimura S and Ishikawa T 1991 Appl. Phys. Lett. 59 2778-2780

[14] Tzimis A, Trifonov A V, Christmann G, Tsintzos S I, Hatzopoulos Z, Ignatiev I V, Kavokin A V and Savvidis P G 2015 Appl. Phys. Lett. 107101101

[15] Kavokin A, Liew T C H, Schneider C and Höfling S 2016 Low Temp. Phys. 42 323-329

[16] Liew T C H, Glazov M M, Kavokin K V, Shelykh I A, Kaliteevski M A and Kavokin A V 2015 Phys. Rev. Lett. 110047402

[17] Trifonov A V, Cherotchenko E D, Carthy J L, Ignatiev I V, Tzimis A, Tsintzos S, Hatzopoulos Z, Savvidis P G and Kavokin A V 2016 Phys. Rev. B 93125304

[18] Miller R C, Kleinman D A and Gossard A C 1984 Phys. Rev. B 29 7085-7087

[19] Aspnes D E, Quinn W E, Tamargo M C, Pudensi M A A, Schwarz S A, Brasil M J S P, Nahory R E and Gregory S 1992 Appl. Phys. Lett. 60 1244-1246

[20] Chu-Liang Y and Qing Y 1988 Phys. Rev. B 37 1364-1367 
[21] Ramirez J M, Vakarin V, Gilles C, Frigerio J, Ballabio A, Chaisakul P, Le Roux X, Alonso-Ramos C, Maisons G, Vivien L, Carras M, Isella G and Marris-Morini D 2017 Optics Lett. 42 105-108

[22] Walther T, Humphreys C and Rodenburg J 1997 Quantification of the composition of silicon germanium/silicon structures by high-angle annular dark field imaging vol 153 (Institute of Physics) pp 303-306

[23] Grieb T, Tewes M, Schowalter M, Müller-Caspary K, Krause F F, Mehrtens T, Hartmann J M and Rosenauer A 2018 Ultramicroscopy 184 29-36

[24] Tewes M, Krause F F, Müller K, Potapov P, Schowalter M, Mehrtens T and Rosenauer A 2013 J. Phys. Conf. Ser. 471012011

[25] Müller-Caspary K, Oppermann O, Grieb T, Krause F F, Rosenauer A, Schowalter M, Mehrtens T, Beyer A, Volz K and Potapov P 2016 Sci. Reports 637146

[26] Isella G, Chrastina D, Rössner B, Hackbarth T, Herzog H J, König U and von Känel H 2004 Solid State Electron. 48 1317-1323

[27] Marchionna S, Virtuani A, Acciarri M, Isella G and von Kaenel H 2006 Mat. Sci. Semicond. Process. 9 802-805

[28] Darwin C G 1914 Phil. Mag. 27 315-333

[29] Morelhão S L, Fornari C I, Rappl P H O and Abramof E 2017 J. Appl. Cryst. 50 $399-410$

[30] Kriegner D, Wintersberger E and Stangl J 2013 J. Appl. Cryst. 46 1162-1170

[31] Jones L, Yang H, Pennycook T J, Marshall M S J, Van Aert S, Browning N D, Castell M R and Nellist P D 2015 Advanced Structural and Chemical Imaging 18

[32] Oelerich J O, Duschek L, Belz J, Beyer A, Baranovskii S D and Volz K 2017 Ultramicroscopy $\mathbf{1 7 7} 91-96$

[33] Duschek L, Kükelhan P, Beyer A, Firoozabadi S, Oelerich J O, Fuchs C, Stolz W, Ballabio A, Isella G and Volz K 2019 Ultramicroscopy 200 84-96 URL https://doi.org/10.1016/j.ultramic.2019.02.009

[34] Persichetti L, Sgarlata A, Fanfoni M and Balzarotti A 2015 J. Phys.: Condens. Matt. 27253001

[35] Birner S, Zibold T, Andlauer T, Kubis T, Sabathil M, Trellakis A and Vogl P 2007 IEEE T. Electron Dev. $\mathbf{5 4}$ 2137-2142

[36] Lever L, Hu Y, Myronov M, Liu X, Owens N, Gardes F Y, Marko I P, Sweeney S J, Ikonić Z, Leadley D R, Reed G T and Kelsall R W 2011 Optics Lett. 36 4158-4160

[37] Isella G, Ballabio A and Frigerio J 2016 Ge/SiGe quantum well for photonic applications: modelling of the quantum confined Stark effect Silicon Photonics and Photonic Integrated Circuits $V$ vol Proc. SPIE 9891 ed Vivien L, Pavesi L and Pelli S p $98910 \mathrm{G}$

[38] Paul D J 2016 J. Appl. Phys. 120043103 
[39] Li Y H, Walsh A, Chen S, Yin W J, Yang J H, Li J, Da Silva J L F, Gong X G and Wei S H 2009 Appl. Phys. Lett. 94212109

[40] Teherani J T, Chern W, Antoniadis D A, Hoyt J L, Ruiz L, Poweleit C D and Menéndez J 2012 Phys. Rev. B 85205308

[41] Wei S H and Zunger A 1999 Phys. Rev. B 60 5404-5411

[42] Van de Walle C G 1989 Phys. Rev. B 39 1871-1883

[43] Laude L D, Pollak F H and Cardona M 1971 Phys. Rev. B 3 2623-2636

[44] Liu J, Cannon D D, Wada K, Ishikawa Y, Danielson D T, Jongthammanurak S, Michel J and Kimerling L C 2004 Phys. Rev. B 70155309

[45] Aulbur W G, Jönsson L and Wilkins J W 2000 Quasiparticle calculations in solids Solid State Physics vol 54 ed Ehrenreich H and Spaepen F (Academic Press) pp $1-218$

[46] Seiler D G and Littler C L 1992 The Spectroscopy of Semiconductors (New York: Academic Press Inc.) ISBN 0127521364

[47] Cardona M 1970 Modulation spectroscopy of semiconductors Festkörperprobleme 10: Plenary Lectures of the Professional Groups "Semiconductor Physics", "Low Temperature Physics", "Thermodynamics", "Metal Physics" of the German Physical Society Freudenstadt, April 6-11, 1970 ed Madelung O (Berlin, Heidelberg: Springer Berlin Heidelberg) pp 125-173

[48] Pollak F H and Shen H 1993 Mater. Sci. Eng. Rep. 10 275-374

[49] Hsu H P, Wu P H, Huang Y S, Chrastina D, Isella G, von Känel H and Tiong K K 2012 Appl. Phys. Lett. 100041905

[50] Hsu H P, Yang P H, Huang J K, Wu P H, Huang Y S, Li C, Huang S H and Tiong K K 2013 Adv. Condens. Matter Phys. 2013298190 